Vol. 47 (1993) [25-40]

\title{
ON MINIMAX INEQUALITIES ON SPACES HAVING CERTAIN CONTRACTIBLE SUBSETS
}

\begin{abstract}
Sehie Park
The concept of a convex space is extended to an $H$-space; that is, a space having certain family of contractible subsets. For such spaces the KKM type theorems, the Fan-Browder fixed point theorem, the Ky Fan type matching theorem, and minimax inequalities are given. Moreover, applications to a von Neumann-Sion type minimax theorem, a saddle point theorem, a quasi-variational inequality, and a Kakutani type fixed point theorem are obtained.
\end{abstract}

\section{INTRODUCTION}

The celebrated Ky Fan minimax inequality [8] has numerous applications in various and diverse fields of mathematics. Also, there have appeared a number of equivalent formulations and generalisations. For the literature, see $[14,16]$.

It is well-known that the Brouwer fixed point theorem, the Sperner lemma, the Knaster-Kuratowski-Mazurkiewicz theorem (simply, KKM theorem), Fan's geometric lemma, the Fan-Browder fixed point theorem, Fan's minimax inequality, a matching theorem, and many others are equivalent. Since their applications are so broad and rich, such area of study can be adequately called the KKM theory.

At first, this theory was mainly concerned with convex subsets of topological vector spaces. However, recently, it has been extended to convex spaces by Lassonde [12], and to pseudo-convex spaces, contractible spaces, or spaces having certain families of contractible subsets (simply, $H$-spaces $[2]$ ) by Horvath $[9,10,11]$. This line of generalisations of earlier works are followed by Bardaro and Ceppitelli [2], Ding and Tan [6], Ding, Kim, and Tan [4, 5], Tarafdar [18], and Park [16]. Influenced by Zhou and Chen [19], some of those authors claimed to obtain new minimax inequalities for $H$-spaces and their applications. However, some of the inequalities were incorrectly stated and mutual relations among them and some basic results in the KKM theory were misstated or ignored.

In the present paper, we introduce two KKM type theorems for $\boldsymbol{H}$-spaces with new coercivity conditions and some equivalent formulations including generalisations of

Received 4th December 1991.

Supported in part by the Basic Sciences Research Institute Program, Ministry of Education, 1992.

Copyright Clearance Centre, Inc. Serial-fee code: 0004-9729/93 SA2.00+0.00. 
known minimax inequalities. Moreover, applications to a von Neumann type minimax theorem, a saddle point theorem, a quasi-variational inequality, and a Kakutani type fixed point theorem are discussed.

In Section 3 we state the KKM type theorems and their basic equivalent formulations for $H$-spaces. Our method is based on that of Tarafdar [18] and Park [16].

Section 4 deals with generalisations of known minimax inequalities for $\boldsymbol{H}$-spaces and the mutual relations among them and the KKM type theorems.

In Section 5 we give some direct applications of our minimax inequalities and their equivalent formulations. We obtain a generalised form of the von Neumann-Sion minimax theorem for $H$-spaces. We also give a generalised version of a saddle point theorem [16, Theorem 2.14] and an improved version of a quasi-variational inequality [19, Theorem 3.1]. Finally, we note that the latter implies a general form of the Kakutani fixed point theorem.

\section{Prelimin aries}

Let $X$ and $Y$ be two sets. A multifunction $F: X \rightarrow 2^{Y}$ is a function from $X$ into the power set $2^{Y}$ of $Y$. Let $F(D)=\bigcup\left\{F_{x}: x \in D\right\}$ for $D \subset X$ and $F^{-1} y=\left\{x \in X: y \in F_{x}\right\}$ for $y \in Y$. Let $\mathcal{F}(D)$ be the family of all nonempty finite subsets of $D$.

A convex space $X$ is a nonempty convex set (in a vector space) with any topology that induces the Euclidean topology on the convex hulls of its finite subsets. For details, see Lassonde [12]. For a nonempty subset $D$ of $X$, a multifunction $F: D \rightarrow 2^{X}$ is said to be $K K M$ if co $A \subset F(A)$ for each $A \in \mathcal{F}(D)$, where co denotes the convex hull.

A subset $C$ of a topological space $X$ is said to be compactly closed [respectively, open] in $X$ if for every compact set $K \subset X$ the set $C \cap K$ is closed [respectively, open] in $K$. A topological space $X$ is said to be contractible if the identity map $1_{X}$ of $X$ is homotopic to a constant map.

Motivated by earlier works of Horvath [9, 10, 11] and Bardaro and Ceppitelli [2], we introduce the following notions.

A triple $(X, D ; \Gamma)$ is called an $H$-space if $X$ is a topological space, $D$ a nonempty subset of $X$, and $\Gamma=\left\{\Gamma_{A}\right\}$ a family of contractible subsets of $X$ indexed by $A \in \mathcal{F}(D)$ such that $\Gamma_{A} \subset \Gamma_{B}$ whenever $A \subset B \in \mathcal{F}(D)$. (The triple is called an $H$-space in [2] whenever $D=X$.) If $D=X$, we denote $(X ; \Gamma)$ instead of $(X, X ; \Gamma)$. For an $H$-space $(X ; \Gamma)$ and any nonempty subset $Y$ of $X$, we have an $H$-space $(X, Y ; \Gamma)$.

Any convex space $X$ is an $H$-space $(X ; \Gamma)$ by putting $\Gamma_{A}=$ co $A$. Other examples of $(X ; \Gamma)$ are any pseudo-convex space [9], any homeomorphic image of a convex space, any contractible space, and so on. For other examples, see [2]. Every $n$-simplex $\Delta_{n}$ is an $H$-space $\left(\Delta_{n}, D ; \Gamma\right)$, where $D$ is the set of vertices and $\Gamma_{A}=\operatorname{co} A$ for $A \in \mathcal{F}(D)$. 
For an $(X, D ; \Gamma)$, a subset $C$ of $X$ is said to be $H$-convex if for each $A \in \mathcal{F}(D)$, $A \subset C$ implies $\Gamma_{A} \subset C$. Note that $X$ itself and $\emptyset$ are $B$-convex. A multifunction $F: D \rightarrow 2^{X}$ is said to be $H-K K M$ if $\Gamma_{A} \subset F(A)$ for each $A \in \mathcal{F}(D)$ [2]. A subset $L$ of $X$ is called an $H$-subspace of $(X, D ; \Gamma)$ if $L \cap D \neq \emptyset$ and for every $A \in \mathcal{F}(L \cap D), \Gamma_{A} \cap L$ is contractible. This is equivalent to saying that the triple $\left(L, L \cap D ;\left\{\Gamma_{A} \cap L\right\}\right)$ is an $H$-space. (An $H$-subspace is called weakly $H$-convex in [2] whenever $X=D$.)

Recently, Tarafdar [18] introduced the concept of an $H$-KKM map more strict than that of [2], but more in line with the usual one in a vector space. Motivated by [18], we introduce the following:

Given a nonempty subset $S$ of an $H$-space $(X, D ; \Gamma)$, the $H$-convex hull of $S$, denoted by $H$-co $S$, is defined by

$$
H \text { - co } S=\bigcap\{Y: S \subset Y \subset X \text { and } Y \text { is } H \text {-convex }\} \text {. }
$$

As in [18], note that $H$-co $S$ is the smallest $H$-convex subset of $X$ containing $S$ and that

$$
H \text { - co } S=\bigcup\{H \text { - co } A: A \in \mathcal{F}(S)\} \text {. }
$$

Following Tarafdar [18], a multifunction $F: D \rightarrow 2^{X}$ is said to be $H-K K M$ (which will be denoted $T$-KKM in this paper) if $H$-co $A \subset F(A)$ for each $A \in \mathcal{F}(D)$. Note that $T$-KKM implies $H$-KKM. In fact, $\Gamma_{A} \subset H$-co $A$ since $A \subset H$-co $A$ and $H$-co $A$ is $H$-convex.

LEMma. Let $(X, D ; \Gamma)$ be an $H$-space and $G: D \rightarrow 2^{X}$ an $H$-KKM multifunction with compactly closed values. Then every finite subfamily of $\{G x: x \in D\}$ has a nonempty intersection.

Lemma is given in [16, Theorem 1] using the KKM theorem and the method of Horvath [10, Theorem 1, 11, Theorem 1].

Recall that an extended real-valued function $f: X \rightarrow \overline{\mathbf{R}}$ on a topological space $X$ is lower [respectively, upper] semicontinuous (l.s.c.) [respectively, u.s.c.] if $\{x \in X$ : $f x>r\}$ [respectively, $\{x \in X: f x<r\}$ ] is open for each $r \in \overline{\mathbf{R}}$.

\section{THE KKM TYPE THEOREMS AND THEIR EQUIVALENCIES}

We begin with the following KKM theorem for $H$-spaces.

THEOREM 1. Let $(X, D ; \Gamma)$ be an $H$-space and $G: D \rightarrow 2^{X}$ an $H$-KKM multifunction with compactly closed values. Suppose that there exists a nonempty compact subset $K$ of $X$ such that either

(i) $\bigcap\{G x: x \in M\} \subset K$ for some $M \in \mathcal{F}(D)$; or

(ii) for each $N \in \mathcal{F}(D)$, there exists a compact $H$-subspace $L_{N}$ of $X$ containing $N$ such that $L_{N} \cap \bigcap\left\{G x: x \in L_{N} \cap D\right\} \subset K$. 
Then $K \cap \bigcap\{G x: x \in D\} \neq \emptyset$.

Proof: Case (i). Clear from the Lemma.

Note that, from Case (i), if $X$ itself is compact, then Theorem 1 holds without assuming (i) or (ii). From this fact we can deduce Case (ii) as follows:

Case (ii). Suppose that $K \cap \cap\{G x: x \in D\}=\emptyset$; that is, $K \subset \bigcup\{X \backslash G x: x \in D\}$. Since the compact subset $K$ is covered by compactly open sets $X \backslash G x, x \in D$, there exists an $N \in \mathcal{F}(D)$ such that $K \subset \bigcup\{X \backslash G x: x \in N\}$. Let $L_{N}$ be the compact $H$-subspace in (ii) and $G^{\prime}: L_{N} \cap D \rightarrow 2^{L_{N}}$ a multifunction defined by $G^{\prime} x=G x \cap L_{N}$ for $x \in L_{N} \cap D$. Then each $G^{\prime} x$ is closed in $L_{N}$. Moreover, $G^{\prime}$ is $H$-KKM. For,

$$
\begin{gathered}
A \in \mathcal{F}\left(L_{N} \cap D\right) \Longrightarrow A \in \mathcal{F}(D) \Longrightarrow \Gamma_{A} \subset G(A) \Longrightarrow \\
\Gamma_{A} \cap L_{N} \subset G(A) \cap L_{N}=G^{\prime}(A) .
\end{gathered}
$$

Therefore, by Case (i), we have

$$
\bigcap_{x \in L_{N} \cap D} G^{\prime} x=L_{N} \cap \bigcap_{x \in L_{N} \cap D} G x \neq 0
$$

Let $z \in L_{N} \cap \cap\left\{G x: x \in L_{N} \cap D\right\}$. If $z \in L_{N} \cap K$, then

$$
z \in K \subset \bigcup_{x \in N}(X \backslash G x)
$$

and hence $z \notin G x$ for some $x \in N \subset L_{N} \cap D$, which is a contradiction. Therefore, we have $z \in L_{N} \backslash K$. This implies $z \notin \bigcap\left\{G x: x \in L_{N} \cap D\right\}$ by (ii), which leads another contradiction. Therefore, we must have $K \cap \cap\{G x: x \in D\} \neq 0$. This completes our proof.

Remarks: 1. If $X$ is a convex space with $\Gamma_{A}=\operatorname{co} A$, then (i) implies (ii). In fact, we can choose $L_{N}=\operatorname{co}(M \cup N)$. However, in general, we cannot say (i) $\Longrightarrow$ (ii) for $\boldsymbol{H}$-spaces.

2. For particular forms or related results to Theorem 1, see the remarks on $[16$, Theorem 1 and 4].

3. As in the generalisation of the KKM theorem due to Ky Fan [7, Lemma 1], (i) can be replaced by

(i)' $G x_{0}$ is compact for some $x_{0} \in D$.

However, (i) $\Rightarrow(i)^{\prime}$. For example, let $X=\mathbf{R}, D=\mathbf{Z}, \Gamma_{A}=$ co $A$, and define $G: \mathbf{Z} \rightarrow 2^{\mathbf{R}}$ by $G(0)=\mathbf{R}, G(n)=(-\infty, n]$, and $G(-n)=[-n,+\infty \geqslant)$ for $n \in \mathbf{N}$.

The following form of argument is used in [4]. Let - denote the closure. 
Corollary 1. Let $(X ; \Gamma)$ be an $H$-space and $F, G: X \rightarrow 2^{X}$ two multifunctions such that

(1) for each $x \in X, F x \subset G x$ and $G x$ is closed; and

(2) $F$ is $H-K K M$.

Suppose that either

(i) $\overline{F x_{0}}$ is compact for some $x_{0} \in X$; or

(ii) there exist a nonempty compact subset $K$ of $X$ and, for each $N \in \mathcal{F}(X)$, a compact $H$-subspace $L_{N}$ of $X$ containing $N$ such that $L_{N} \cap \cap\left\{\overline{F_{x}}: x \in L_{N}\right\} \subset K$.

Then

$$
\bigcap\{G x: x \in X\} \neq \emptyset \text {. }
$$

Proof: Since $\overline{F x} \subset G x$ and (2) implies that $\bar{F}$ is $H$-KKM, by Theorem 1, we have

$$
\bigcap\{\overline{F x}: x \in X\} \neq \emptyset \text {. }
$$

This completes our proof.

Since $T$-KKM implies $H$-KKM, from Theorem 1, we have the following:

TheOREM 2. Let $(X, D ; \Gamma)$ be an $H$-space and $G: D \rightarrow 2^{X}$ a multifunction such that

(1) for each $x \in D, G x$ is compactly closed; and

(2) $G$ is $T-K K M$.

Suppose that there exists a nonempty compact subset $K$ of $X$ such that either (i) or (ii) of Theorem 1 holds. Then

$$
K \cap \bigcap\{G: x \in D\} \neq \emptyset .
$$

Theorem 2 has some equivalent formulations. The following is a whole intersection theorem.

Theorem 3. Let $(X, D ; \Gamma)$ be an $H$-space and $F: X \rightarrow 2^{X}, G: D \rightarrow 2^{X}$ multifunctions such that

(1) for each $x \in D, F x \subset G x$ and $G x$ is compactly closed;

(2) for each $y \in X, X \backslash F^{-1} y$ is $H$-convex; and

(3) for each $x \in X, x \in F x$.

Suppose that there exists a nonempty compact subset $K$ of $X$ such that either (i) or (ii) of Theorem 1 holds. Then

$$
K \cap \bigcap\{G x: x \in D\} \neq \emptyset .
$$


Proof: We show that (2) and (3) imply that $G$ is $T$-KKM. Suppose that there exists an $A \in \mathcal{F}(D)$ such that $H$-co $A \not \subset G(A)$; that is, there exists a $y \in H$-co $A$ such that $y \notin G x$ for all $x \in A$. In other words, $A \subset X \backslash G^{-1} y \subset X \backslash F^{-1} y$. Since $X \backslash F^{-1} y$ is $H$-convex by (2) and contains $A$, we have $y \in H$-co $A \subset X \backslash F^{-1} y$, which contradicts (3). Therefore, by Theorem 2 , we have the conclusion.

REMARK: For related results to Theorem 3, see [16, Theorem 7].

From Theorem 3 we obtain the following Fan-Browder type fixed point theorem or a maximal element theorem.

Theorem 4. Let $(X, D ; \Gamma)$ be an $H$-space and $S: D \rightarrow 2^{X}, T: X \rightarrow 2^{X}$ multifunctions such that

(1) for each $x \in D, S x \subset T x$ and $S x$ is compactly open; and

(2) for each $y \in X, T^{-1} y$ is $H$-convex.

Suppose that there exists a nonempty compact subset $K$ of $X$ such that either

(i) $X \backslash K \subset S(M)$ for some $M \in \mathcal{F}(D)$; or

(ii) for each $N \in \mathcal{F}(D)$, there exists a compact $H$-subspace $L_{N}$ of $X$ containing $N$ such that $L_{N} \backslash K \subset S\left(L_{N} \cap D\right)$.

Then either there exists a $y_{0} \in K$ such that $S^{-1} y_{0}=\emptyset$ or there exists an $x_{0} \in X$ such that $x_{0} \in T x_{0}$.

Proof: Suppose that $x \notin T x$ for each $x \in X$. Let $F x=X \backslash T x$ for $x \in X$ and $G x=X \backslash S x$ for $x \in D$. Then all the requirements of Theorem 3 are satisfied. Therefore there exists a $y_{0} \in K \cap \bigcap\{G x: x \in D\}$; that is, $y_{0} \notin S x$ for all $x \in D$. In other words, $S^{-1} y_{0}=\emptyset$. This completes our proof.

REMARKS: 1 . The $y_{0}$ in the conclusion of Theorem 4 is called a maximal element in the sense that $x \leqslant y$ if and only if $x \in S y$.

2. The equivalency of less general versions of Theorems 2 and 4 was given by Tarafdar [18].

3. For related results to Theorem 4, see the remarks on [16, Theorem 6].

From Theorem 4, we obtain the following Ky Fan type matching theorem for open coverings of an $H$-space.

ThEOREM 5. Let $(X, D ; \Gamma)$ be an $H$-space and $S: D \rightarrow 2^{X}$ a multifunction such that

(1) for each $x \in D, S x$ is compactly open.

Suppose that there exists a nonempty compact subset $K$ of $X$ such that

(2) $K \subset S(D)$

and that either (i) or (ii) of Theorem 4 holds. 
Then there exists an $A \in \mathcal{F}(D)$ such that

$$
(B-\operatorname{co} A) \cap \bigcap\{S x: x \in A\} \neq 0 .
$$

Proof: For each $y \in X$, put $T^{-1} y=H$-co $\left(S^{-1} y\right)$. This defines a multifunction $T: X \rightarrow 2^{X}$. Then all the requirements of Theorem 4 are satisfied. In fact, for each $x \in D, x \in S^{-1} y$ for some $y \in X$ implies $x \in T^{-1} y$; that is, $S x \subset T x$. Moreover, $T^{-1} y$ is $H$-convex for each $y \in X$. Further, $S^{-1} y \neq \emptyset$ for each $y \in K$ by (2). Therefore, by Theorem 4, there exists an $x_{0} \in X$ such that $x_{0} \in T x_{0}$ or $x_{0} \in T^{-1} x_{0}$. By the definition of $T^{-1} x_{0}$, we have an $A \in \mathcal{F}\left(S^{-1} x_{0}\right)$ such that $x_{0} \in H$-co $A$. Note that $S^{-1} x_{0} \subset D$ and hence $A \in \mathcal{F}(D)$. Since $x_{0} \in S x$ for all $x \in A$, we have $x_{0} \in(H-\operatorname{co} A) \cap \bigcap\{S x: x \in A\}$. This completes our proof.

In fact, Theorems 2-5 are equivalent.

Proof of Theorem 2 using Theorem 5: Let $S x=X \backslash G x$ for each $x \in D$. Suppose that $K \cap \bigcap\{G x: x \in D\}=\emptyset$, or equivalently, $S(D) \supset K$. Then, by Theorem 5, there exists an $A \in \mathcal{F}(D)$ such that

$$
(H-\operatorname{co} A) \cap \bigcap\{S x: x \in A\} \neq \emptyset \text { or } \quad H \text { - } \operatorname{co} A \not \subset G(A) \text {. }
$$

This contradicts the fact that $G$ is $T$-KKM. This completes our proof.

REMARKs: 1 . If the conclusion of Theorem 5 is replaced by the existence of $A \in$ $\mathcal{F}(D)$ satisfying $\Gamma_{A} \cap \bigcap\{S x: x \in A\} \neq 0$, then we obtain an equivalent form of Theorem 1. Therefore, for a convex space $X$ with $\Gamma_{A}=H$-co $A=\operatorname{co} A$, Theorem 1 is also equivalent to any of Theorems 2-5.

2. For related results to Theorem 5 , see [16, Theorem 5].

\section{MinimaX INEQUalities}

In this section, we show that the KKM theorem and the whole intersection theorem are equivalent to minimax inequalities.

The following is equivalent to Theorem 1.

Theorem 6. Let $(X, D ; \Gamma)$ be an $H$-space, $\phi: D \times X \rightarrow \overline{\mathbf{R}}$ an extended realvalued function, and $\gamma \in \overline{\mathbf{R}}$ such that

(1) for each $x \in D,\{y \in X: \phi(x, y) \leqslant \gamma\}$ is compactly closed; and

(2) for each $N \in \mathcal{F}(D)$ and each $y \in \Gamma_{N}, \min _{x \in N} \phi(x, y) \leqslant \gamma$.

Suppose that there exists a nonempty compact subset $K$ of $X$ such that either

(i) there exists an $M \in \mathcal{F}(D)$ such that

$$
\{y \in X: \phi(x, y) \leqslant \gamma \text { for all } x \in M\} \subset K \text {; or }
$$


(ii) for each $N \in \mathcal{F}(D)$, there exists a compact $B$-subspace $L_{N}$ of $X$ containing $N$ such that for each $y \in L_{N} \backslash K$ there exists an $x \in L_{N} \cap D$ satisfying $\phi(x, y)>\gamma$.

Then there exists a $\bar{y} \in K$ such that

$$
\sup _{x \in D} \phi(x, \bar{y}) \leqslant \gamma .
$$

Proof of Theorem 6 using Theorem 1: Let $G x=\{y \in X: \phi(x, y) \leqslant \gamma\}$ for each $x \in D$. Then $G$ has compactly closed values and the coercivity condition (i) or (ii) of Theorem 1 holds. It remains to show that $G$ is $H$-KKM. Suppose that there exists an $N \in \mathcal{F}(D)$ such that $\Gamma_{N} \not \subset G(N)$; that is, there exists a $y \in \Gamma_{N}$ such that $y \notin G x$ or equivalently $\phi(x, y)>\gamma$ for all $x \in N$. Then $\min _{x \in N} \phi(x, y)>\gamma$, which contradicts (2). By Theorem 1, there exists a $\bar{y} \in K \cap \bigcap\{G x: x \in D\}$; that is, $\phi(x, \bar{y}) \leqslant \gamma$ for all $x \in D$. This completes our proof.

Proof of Theorem 1 using Theorem 6: Define $\phi: D \times X \rightarrow \mathbf{R}$ by

$$
\phi(x, y)= \begin{cases}0 & \text { if } \quad y \in G x \\ 1 & \text { otherwise }\end{cases}
$$

for $(x, y) \in D \times X$. Put $\gamma=0$ in Theorem 6 . Then (1) holds since $G$ has compactly closed values. Moreover, the coercivity condition (i) or (ii) of Theorem 6 holds. Further, (2) holds since $G$ is $H$-KKM. In fact, suppose that there exist an $N \in \mathcal{F}(D)$ and $y \in \Gamma_{N}$ such that $\min _{x \in N} \phi(x, y)>0$. Then $\phi(x, y)>0$ for all $x \in N$; that is, $y \notin G(N)$, which is a contradiction. Therefore, by Theorem 6 , there exists a $\bar{y} \in K$ such that $\phi(x, \bar{y})=0$ for all $x \in D$; that is, $\bar{y} \in \bigcap\{G x: x \in D\}$. This completes our proof.

REMARKS: 1. Condition (1) is implied by the following:

(1)' for each $x \in D, y \mapsto \phi(x, y)$ is l.s.c. on any compact subset of $X$.

2. It is shown that, in [4], (2) is equivalent to the condition that $G$ is $H-\mathrm{KKM}$ as in the above proofs. For a convex space $X$ with $\Gamma_{A}=$ co $A$ and $X=D,(2)$ is called the $\gamma$-diagonal quasi-convexity (simply, $\gamma$-DQCV) of $\phi(x, y)$ in $x$, by Zhou and Chen [19].

3. The coercivity condition (i) is implied by the following:

(i)' for some $x_{0} \in D, y \mapsto \phi\left(x_{0}, y\right)$ is inf-compact.

4. Theorem 6 is a correct version of Ding and Tan [6, Theorem 16].

5. If $X=K$ itself is compact, then Theorem 6 holds without assuming the coercivity conditions (i) and (ii). For a compact convex space $X$, Theorem 6 with $D=X$ extends Zhou and Chen [19, Theorem 2.11], whose proof is based on the partition of unity argument and the Brouwer fixed point theorem. 
COROLlary 6.1. Under the hypothesis of Theorem 6 , if $\gamma=\sup _{x \in D} \phi(x, x)$, then there exists a $\bar{y} \in K$ such that

$$
\sup _{x \in D} \phi(x, \bar{y}) \leqslant \sup _{x \in D} \phi(x, x)
$$

REMARK: For a compact convex space $X$, Corollary 6.1 with $D=X$ extends Zhou and Chen [19, Corollary 2.12].

Corollary 6.2. Let $(X ; \Gamma)$ be an $H$-space, and $\phi, \psi: X \times X \rightarrow \overline{\mathbf{R}}$ multifunctions satisfying

(1) $\phi(x, y) \leqslant \psi(x, y)$ for each $(x, y) \in X \times X$;

(2) for each $x \in X, y \mapsto \phi(x, y)$ is l.s.c. on $X$; and

(3) the map $x \mapsto F x=\{y \in X: \psi(x, y) \leqslant 0\}$ is $H$-KKM.

Suppose that either (i) or (ii) of Corollary 1 holds. Then there exists a $\bar{y} \in X$ such that

$$
\sup _{x \in X} \phi(x, \bar{y}) \leqslant 0 .
$$

Proof: Let $G x=\{y \in X: \phi(x, y) \leqslant 0\}$ for each $x \in X$. Note that $F x \subset G x$ for each $x \in X$ by (1), and $G x$ is closed by (2). This shows that $\bar{F}$ has closed values and (3) implies that $\bar{F}$ is $H$-KKM. Therefore, from Corollary 1 or Theorem 6 , we have the conclusion.

REMARKS: 1 . The coercivity condition (i) of Corollary 1 can be replaced by the following:

(i)' there exist a nonempty closed compact subset $K$ of $X$ and an $x_{0} \in X$ such that $\psi\left(x_{0}, y\right)>0$ for all $y \in X \backslash K$.

In fact, from (i)', we have $F x_{0} \subset K$ so that $\overline{F x_{0}} \subset \bar{K}=K$, and hence $\overline{F x_{0}}$ is compact.

2. Ding, Kim, and Tan [4, Theorems 1 and 2] obtained Corollary 6.2 for Case (i)' under an additional condition that $\psi(x, x) \leqslant 0$ for each $x \in X$. This assumption is superfluous. Influenced by this, many of the other results in $[4,5]$ are incorrectly stated. For example, the correct form of $[4$, Theorem 5$]$ is our Theorem 3. Moreover, the hypothesis of $[4$, Theorem 5$]$ is self-contradictory.

3. A generalisation of Corollary 6.2 for Case (ii) is given by Park [16, Theorem 10].

Theorem 3 also can be reformulated as a minimax inequality.

TheOREM 7. Let $(X, D ; \Gamma)$ be an $H$-space, $\phi: D \times X \rightarrow \overline{\mathbf{R}}, \psi: X \times X \rightarrow \overline{\mathbf{R}}$ functions, and $\gamma \in \overline{\mathrm{R}}$ such that

(1) $\phi(x, y) \leqslant \psi(x, y)$ for all $(x, y) \in D \times X$ and $\psi(x, x) \leqslant \gamma$ for all $x \in X$;

(2) for each $x \in D,\{y \in X: \phi(x, y) \leqslant \gamma\}$ is compactly closed; and

(3) for each $y \in X,\{x \in X: \psi(x, y)>\gamma\}$ is $H$-convex. 
Suppose that there exists a nonempty compact subset $K$ of $X$ such that either (i) or (ii) of Theorem 6 holds. Then there exists a $\bar{y} \in K$ such that

$$
\sup _{x \in D} \phi(x, \bar{y}) \leqslant \gamma
$$

Proof of Theorem 7 using Theorem 3: Put $F x=\{y \in X: \psi(x, y) \leqslant \gamma\}$ for $x \in X$ and $G x=\{y \in X: \phi(x, y) \leqslant \gamma\}$ for $x \in D$. Then Theorem 7 follows from Theorem 3. Note that Theorem 7 also follows from Theorem 6 by following the proof of Theorem 3 using Theorem 2.

Proof of Theorem 3 using Theorem 7: Define functions $\phi: D \times X \rightarrow \mathbf{R}$ and $\psi: X \times Y \rightarrow \mathbf{R}$ by

$$
\phi(x, y)= \begin{cases}0 & \text { if } \quad y \in G x \\ 1 & \text { otherwise }\end{cases}
$$

for $(x, y) \in D \times X$ and

$$
\psi(x, y)= \begin{cases}0 & \text { if } y \in F x \\ 1 & \text { otherwise }\end{cases}
$$

for $(x, y) \in X \times X$. Then $\phi(x, y) \leqslant \psi(x, y)$ for all $(x, y) \in D \times X$ since $F x \subset G x$ for $x \in D$, and $\psi(x, x)=0$ for all $x \in X$ since $x \in F x$. Other requirements of Theorem 7 are satisfied for $\gamma=0$. Therefore, there exists a $\bar{y} \in K$ such that $\phi(x, \bar{y})=0$ for all $x \in D$; that is, $\bar{y} \in G x$ for all $x \in D$. This completes our proof.

REMARKs: 1 . Since Theorem 1 generalises Theorem 3, we know that Theorem 6 generalises Theorem 7. However, for convex spaces those are all equivalent.

2. Ding, Kim, and Tan [4] gave examples of convex spaces showing that particular forms of Corollary 6.2 for Case (i) and Theorem 7 for Case (ii) are independent. However, for convex spaces, those two results are included in Theorem 7 for Case (ii).

3. Theorem 7 for Case (ii) is a correct form of Ding and Tan [6, Theorem 17]. For other related results to Theorem 7 for Case (ii), see the remarks on Park [16, Theorem 9].

4. Bardaro and Ceppitelli [2, Theorems 3 and 4] are equivalent to particular forms of Theorem 7 for Case (ii) using a topological Riesz space instead of $\overline{\mathbf{R}}$.

COROLLARY 7. Under the hypothesis of Theorem 7 , if $\gamma=\sup _{x} \in X \psi(x, x)$, then there exists a $\bar{y} \in K$ such that

$$
\sup _{x \in D} \phi(x, \bar{y}) \leqslant \sup _{x \in X} \psi(x, x)
$$


REMARK: For a compact convex space $X$, Corollary 7 with $D=X$ extends Ky Fan's minimax inequality [8, Theorem 1].

Note that the minimax inequalities can be formulated equivalently to analytic alternatives, geometric forms, fixed point versions, or maximal element versions. Moreover, such inequalities have applications to systems of convex inequalities, sets with $H$-convex sections, the von Neumann type minimax theorems, and many others just following [4, 5] (of course, with some necessary corrections).

\section{MinimaX, QUASI-VARIATIONAL INEQUALITY, AND FIXED POINT THEOREMS}

In this section, we first obtain a generalised von-Neumann-Sion minimax theorem from Theorem 4, and then a generalisation of a saddle point theorem due to Zhou and Chen [19, Theorem 2.14]. Moreover, we state an improved version of a quasi-variational inequality [19, Theorem 3.1]. Finally, we note that the latter implies a general form of the Kakutani fixed point theorem.

Theorem 8. Let $X$ and $Y$ be $H$-spaces and $f, g, s, t: X \times Y \rightarrow \overline{\mathbf{R}}$ functions such that

(1) $f \leqslant s \leqslant t \leqslant g$ on $X \times Y$;

(2) for each $x \in X, f(x, \cdot)$ is l.s.c. on $Y$;

(3) for each $y \in Y, g(\cdot, y)$ is u.s.c. on $X$;

(4) for each $y \in Y$ and $c \in R,\{x \in X: s(x, y)>c\}$ is $H$-convex; and

(5) for each $x \in X$ and $c \in \mathbf{R},\{y \in Y: t(x, y)<c\}$ is $H$-convex.

Suppose that there exists a nonempty compact subset $K$ of $X \times Y$ such that for each $c \in \mathbf{R}$, either

(i) there exists an $M \in \mathcal{F}(X \times Y)$ satisfying

$$
(X \times Y) \backslash K \subset \bigcup_{\left(x_{i}, y_{i}\right) \in M}\left\{\bar{x} \in X: g\left(\bar{x}, y_{i}\right)<c\right\} \times\left\{\bar{y} \in Y: f\left(x_{i}, \bar{y}\right)>c\right\}
$$

or

(ii) for each $N \in \mathcal{F}(X \times Y)$, there exists a compact $H$-subspace $L_{N}$ of $X \times Y$ containing $N$ such that

$$
L_{N} \backslash K \subset \bigcup_{(x, y) \in L_{N}}\{\bar{x} \in X: g(\bar{x}, y)<c\} \times\{\bar{y} \in Y: f(x, \bar{y})>c\} .
$$

Then we have

$$
\inf _{y \in Y} \sup _{x \in X} f(x, y) \leqslant \sup _{x \in X} \inf _{y \in Y} g(x, y)
$$


Proof: Suppose that there exists a $c \in \mathbf{R}$ such that

$$
\sup _{x \in X} \inf _{y \in Y} g(x, y)<c<\inf _{y \in Y} \sup _{x \in X} f(x, y) \text {. }
$$

Define multifunctions $S, T: X \times Y \rightarrow 2^{X \times Y}$ by

$$
\begin{aligned}
& S(x, y)=\{\bar{x} \in X: g(\bar{x}, y)<c\} \times\{\bar{y} \in Y: f(x, \bar{y})>c\} \\
& T(x, y)=\{\bar{x} \in X: t(\bar{x}, y)<c\} \times\{\bar{y} \in Y: s(x, \bar{y})>c\}
\end{aligned}
$$

for $(x, y) \in X \times Y$. Note that $S(x, y) \subset T(x, y)$ by $(1)$; and each $S(x, y)$ is open by (2) and (3). Moreover, each fiber

$$
T^{-1}(\bar{x}, \bar{y})=\{x \in X: s(x, \bar{y})>c\} \times\{y \in Y: t(\bar{x}, y)<c\}
$$

is $H$-convex by (4) and (5). Further, the coercivity conditions (i) and (ii) can be written as follows:

(i) $(X \times Y) \backslash K \subset S(M)$ for some $M \in \mathcal{F}(X \times Y)$,

(ii) $L_{N} \backslash K \subset S\left(L_{N}\right)$ for each $N \in \mathcal{F}(X \times Y)$.

Therefore, by Theorem 4, either there exists an $\left(x_{0}, y_{0}\right) \in K$ such that $S^{-1}\left(x_{0}, y_{0}\right)$ $=\emptyset$ or there exists an $\left(x_{0}, y_{0}\right) \in X \times Y$ such that $\left(x_{0}, y_{0}\right) \in T\left(x_{0}, y_{0}\right)$.

However, $S^{-1}\left(x_{0}, y_{0}\right)=\emptyset$ implies $f\left(x, y_{0}\right) \leqslant c$ for all $x \in X$ or $g\left(x_{0}, y\right) \geqslant c$ for all $y \in Y$. This contradicts the assumption on $c$. On the other hand, $\left(x_{0}, y_{0}\right) \in T\left(x_{0}, y_{0}\right)$ implies

$$
c<s\left(x_{0}, y_{0}\right) \leqslant t\left(x_{0}, y_{0}\right)<c,
$$

another contradiction. This completes our proof.

REMARKS: 1 . If $X$ and $Y$ are $H$-spaces, so is $X \times Y$; and the product of $H$-convex sets is $H$-convex [18].

2. If $f=g$ in Theorem 8 , we have the conclusion

$$
\inf _{y \in Y} \sup _{x \in X} f(x, y)=\sup _{x \in X} \inf _{y \in Y} f(x, y) .
$$

Further, if $X$ and $Y$ are compact, then we have the conclusion

$$
\min _{y \in Y} \max _{x \in X} f(x, y)=\max _{x \in X} \min _{y \in Y} f(x, y),
$$

and hence, Theorem 8 extends the von Neumann-Sion minimax theorem for convex spaces [17].

3. Instead of any $c \in \mathbf{R}$ in (4), (5) and (i), (ii) of Theorem 8, we may consider any $c \in \mathbf{R}$ satisfying $c>\sup _{x \in X} \inf _{y \in Y} g(x, y)$.

For convex spaces, we have the following 
Corollary 8. Let $X$ and $Y$ be convex spaces and $f, g, s, t: X \times Y \rightarrow \overline{\mathbf{R}}$ functions satisfying (1)-(5) of Theorem 8 . If one of $X$ and $Y$ is compact, then we have

$$
\inf _{y \in Y} \sup _{x \in X} f(x, y) \leqslant \sup _{x \in X} \inf _{y \in Y} g(x, y) .
$$

Proof: (a) If both $X$ and $Y$ are compact, then the coercivity condition (i) or (ii) holds automatically. Therefore, the conclusion follows.

(b) Suppose that $X$ is compact and

$$
\sup _{x \in X} \inf _{y \in Y} g(x, y)<c<\inf _{y \in Y} \sup _{x \in X} f(x, y)
$$

for some $c \in \mathbf{R}$. Then there exists an $N \in \mathcal{F}(Y)$ such that for any $x \in X$ there exists a $y \in N$ with $g(x, y)<c$. Taking $g^{\prime}=g \mid(X \times$ co $N)$, we get

$$
\sup _{x \in X} \inf _{y \in \operatorname{co} N} g^{\prime}(x, y)<c<\inf _{y \in \operatorname{co}} \sup _{x \in X} f(x, y)
$$

which contradicts Case (a).

REMARKS: 1 . Since $\inf _{\nu \in Y} g(x, y)$ is u.s.c. on $X$, if $X$ is compact, the conclusion of Corollary 8 is actually

$$
\inf _{y \in Y} \sup _{x \in X} f(x, y) \leqslant \max _{x \in X} \inf _{y \in Y} g(x, y) .
$$

Note that for $f=s$ and $t=g$, Corollary 8 reduces to Liu [13, Theorem 1]. In [13], Liu applied Corollary 8 to obtain systems of inequalities, a sup inf sup inequality, the Browder variational inequality, and to prove the Tychonoff fixed point theorem.

2. For the case $X$ and $Y$ are compact, Corollary 8 is due to Ben-El-Mechaiekh, Deguire, and Granas [3, Théoréme 5.4 and Corollaire 5.5]. For $f=g$, this case includes the von Neumann minimax theorem due to Sion [17, Theorem 3.4 and Corollary 3.5].

The following consequence of Theorem 6 is a special form of saddle point theorem.

Theorem 9. Let $(X ; \Gamma)$ be an $H$-space, $\phi: X \times X \rightarrow \overline{\mathbf{R}}$ a function, and $\gamma \in \overline{\mathbf{R}}$ such that

(1) for each $x \in X, \phi(x, \cdot)$ is l.s.c. on compact subsets of $X$;

(2) for each $A \in \mathcal{F}(X)$ and each $y \in \Gamma_{A}, \min _{x \in A} \phi(x, y) \leqslant \gamma$;

(3) there exists a nonempty compact subset $K$ of $X$ satisfying (i) or (ii) of Theorem 6;

$(1)^{\prime}$ for each $y \in X, \phi(\cdot, y)$ is u.s.c. on compact subsets of $X$;

(2)' for each $B \in \mathcal{F}(X)$ and each $x \in \Gamma_{B}, \max _{y \in B} \phi(x, y) \geqslant \gamma$; and 
(3)' there exists a nonempty compact subset $K^{\prime}$ of $X$ satisfying either

(i)' there exists an $M \in \mathcal{F}(X)$ such that

$$
\{x \in X: \phi(x, y) \geqslant \gamma \text { for all } y \in M\} \subset K^{\prime} ;
$$

or

(ii)' for each $N \in \mathcal{F}(X)$, there exists a compact $H$-subspace $L_{N}$ of $X$ containing $N$ such that for each $x \in L_{N} \backslash K^{\prime}$ there exists a $y \in L_{N} \cap X$ satisfying $\phi(x, y)<\gamma$.

Then we have

$$
\max _{x \in K^{\prime}} \inf _{y \in X} \phi(x, y)=\min _{y \in K} \sup _{x \in X} \phi(x, y)=\gamma .
$$

Proof: Apply Theorem 6 to $\phi(x, y)$ with $\gamma$. Then (1)-(3) imply the existence of some $\bar{y} \in K$ such that

$$
\phi(x, \bar{y}) \leqslant \gamma \text { for all } x \in X .
$$

Apply Theorem 6 to $\psi(y, x) \equiv-\phi(x, y)$ with $-\gamma$. Then $(1)^{\prime}-(3)^{\prime}$ imply the existence of some $\bar{x} \in K^{\prime}$ such that

that is,

$$
\begin{array}{rll}
\psi(y, \bar{x}) \leqslant-\gamma & \text { for all } & y \in X \\
\gamma \leqslant \phi(\bar{x}, y) & \text { for all } & y \in X
\end{array}
$$

Therefore, $(\bar{x}, \bar{y}) \in K^{\prime} \times K$ is a saddle point satisfying

$$
\phi(x, \bar{y}) \leqslant \phi(\bar{x}, \bar{y})=\gamma \leqslant \phi(\bar{x}, y)
$$

for all $(x, y) \in X \times X$. This completes our proof.

REMARKS: 1 . In view of Theorem 7, Condition (2) can be replaced by the following without affecting the conclusion of Theorem 9 :

$(2)^{\prime \prime} \phi(x, x) \leqslant \gamma$ for all $x \in X$ and, for each $y \in X,\{x \in X: \phi(x, y)>\gamma\}$ is $H$-convex.

2. If $X$ is a compact convex space, Theorem 9 generalises Zhou and Chen [19, Theorem 2.14], which has potential applications to $N$ person nonzero sum games.

From Theorem 6, we have the following existence theorem for a quasi-variational inequality.

THEOREM 10. Let $K$ be a compact convex subset of a real topological vector space $E$ on which its topological dual $E^{*}$ separates points, and $F: K \rightarrow 2^{K}$ a multifunction with nonempty closed convex values such that

(1) for each $p \in E^{*},\{x \in K: \sup p(F x) \geqslant p(x)\}$ is closed. 
Let $f: K \times K \rightarrow \overline{\mathbf{R}}$ satisfying

(2) for each $y \in K, x \mapsto f(x, y)$ is l.s.c.; and

(3) $f(x, y)$ is $0-D C V$ in $y$; that is, for each $\left\{y_{1}, y_{2}, \cdots, y_{m}\right\} \in \mathcal{F}(K)$ and each $y_{0}=\sum_{i=1}^{m} \alpha_{i} y_{i}\left(\alpha_{i} \geqslant 0, \sum_{i=1}^{m} \alpha_{i}=1\right)$, we have $\sum_{i=1}^{m} \alpha_{i} f\left(y_{0}, y_{i}\right) \geqslant 0$.

Suppose that $F$ and $f$ are related by

(4) $\left\{x \in K: \sup _{y \in F z} f(x, y) \leqslant 0\right\}$ is closed.

Then there exists a solution $\bar{x} \in K$ to the quasi-variational inequality:

$$
\bar{x} \in F \bar{x} \quad \text { and } \sup _{y \in F \bar{x}} f(\bar{x}, y) \leqslant 0 .
$$

Proof: Just follow the proof of [19, Theorem 3.1].

Remarks: 1. Theorem 10 is essentially due to Zhou and Chen [19, Theorem 3.1], which extends [1, Theorem 6.4.21].

2. The class of multifunctions $F: K \rightarrow 2^{K}$ satisfying Condition (1) properly contains that of upper hemicontinuous ones. See [15].

3. If $F=1_{X}$, the identity function of $X$, then Theorem 10 reduces to a particular form of a minimax inequality.

Finally, by putting $f \equiv 0$ in Theorem 10 , we have the following Kakutani type fixed point theorem.

ThEOREM 11. Let $K$ be a compact convex subset of a real topological vector space $E$ on which $E^{*}$ separates points. Then any multifunction $F: K \rightarrow 2^{K}$ satisfying Condition (1) of Theorem 10 with nonempty closed convex values has a fixed point.

REMARKs: 1 . If $F$ is single-valued, Theorem 11 extends earlier works of Brouwer (1910), Schauder (1930), Tychonoff (1935), and Fan (1964), and for multifunctions, Kakutani (1941), Bchnenblust and Karlin (1950), Fan (1952), Glicksberg (1952), and Granas and Liu (1986). For the literature, see [15].

2. Recently, the author [15] established far-reaching generalisations of Theorem 11.

3. Since we obtained Theorem 1 with the aid of an equivalent form of the Brouwer fixed point theorem, Theorems 1-8 and 10-11 are all equivalent, in a wide sense, to the Brouwer theorem.

\section{REFERENCES}

[1] J.-P. Aubin and I. Ekeland, Applied nonlinear analysis (John Wiley and Sons, New York, 1984). 
[2] C. Bardaro and R. Ceppitelli, 'Some further generalizations of Knaster-KuratowskiMazurkiewicz theorem and minimax inequalities', J. Math. Anal. Appl. 132 (1988), 484-490.

[3] H. Ben-El-Mechaiekh, P. Deguire, and A. Granas, 'Points fixes et coincidences pour les applications multivoque II', C.R. Acad. Sci. Paris 295 (1982), 381-384.

[4] X.P. Ding, W.K. Kim, and K.-K. Tan, 'A new minimax inequality on $H$-spaces with applications', Bull. Austral. Math. Soc. 41 (1990), 457-473.

[5] X.P. Ding, W.K. Kim, and K.-K. Tan, 'Applications of a minimax inequality on $H$-spaces', Bull. Austral. Math. Soc. 41 (1990), 475-485.

[6] X.P. Ding and K.-K. Tan, 'Matching theorems, fixed point theorems, and minimax inequalities without convexity', J. Austral. Math. Soc. Ser. A 49 (1990), 111-128.

[7] Ky Fan, 'A generalization of Tychonoff's fixed point theorem', Math. Ann. 142 (1961), 305-310.

[8] Ky Fan, 'A minimax inequality and applications', in Inequalities III, Editor O. Shisha (Academic Press, New York, 1972), pp. 103-113.

[9] C. Horvath, 'Points fixes et coincidences pour les applications multivoques sans convexité', C. R. Acad. Sci. Paris 296 (1983), 403-406.

[10] C. Horvath, 'Points fixes et coïncidences dans les espaces topologiques compacts contractiles', C. R. Acad. Sci. Paris 299 (1984), 519-521.

[11] C. Horvath, 'Some results on multivalued mappings and inequalities without convexity', in Nonlinear and convex analysis, Editors B.L. Lin and S. Simons (Marcel Dekker, New York, 1987), pp. 99-106.

[12] M. Lassonde, 'On the use of KKM multifunctions in fixed point theory and related topics', J. Math. Anal. Appl. 97 (1983), 151-201.

[13] F.-C. Liu, 'A note on the von Neumann-Sion minimax principle', Bull. Inst. Math. Acad. Sinica 6 (1978), 517-524.

[14] S. Park, 'Generalized Fan-Browder fixed point theorems and their applications', in Col lection of Papers Dedicated to J. G. Park on His Sixtieth Birthday (Chunpook National University, 1989), pp. 51-77.

[15] S. Park, 'Fixed point theory of multifunctions in topological vector spaces', J. Korean Math. Soc. 29 (1992), 191-208.

[16] S. Park, 'On the KKM type theorems on spaces having certain contractible subsets', Kyungpook Math. J. (to appear).

[17] M. Sion, 'On general minimax theorems', Pacific J. Math. 8 (1958), 171-176.

[18] E.Tarafdar, 'A fixed point theorem in $H$-space and related results', Bull. Austral. Math. Soc. 42 (1990), 133-140.

[18] J.X. Zhou and G. Chen, 'Diagonal convexity conditions for problems in convex analysis and quasi-variational inequalities', J. Math. Anal. Appl. 132 (1988), 213-225.

Department of Mathematics

Seoul National University

Seoul 151-742

Korea and

The Mathematical Sciences

Research Institute of Korea

Seoul 151-742

Korea 